\title{
Variable anterior segment dysgenesis and cardiac anomalies caused by a novel truncating variant of FOXC1
}

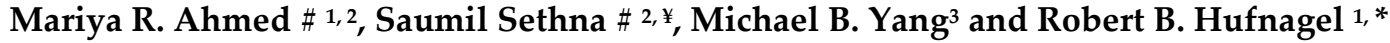

1 Medical Genetics and Ophthalmic Genomics Unit, National Eye Institute, National Institutes of Health, Bethesda, Maryland, USA.; mariya.ahmed@nih.gov \& robert.hufnagel@nih.gov

2 Department of Otorhinolaryngology - Head \& Neck Surgery, University of Maryland School of Medicine, Baltimore, Maryland, USA; SSethna@its.jnj.com

3 Abrahamson Pediatric Eye Institute, Division of Pediatric Ophthalmology, Cincinnati Children's Hospital Medical Center, Department of Ophthalmology, University of Cincinnati College of Medicine, Cincinnati, OH, USA; michael.yang@cchmc.org

¥ Current address: Janssen Pharmaceuticals, South San Francisco, CA

* Correspondence: robert.hufnagel@nih.gov; R.B.H.

\# Contributed equally

\begin{abstract}
Anterior segment dysgenesis (ASD) encompasses a wide spectrum of developmental abnormalities of the anterior ocular segment, including congenital cataract, iris hypoplasia, aniridia, iridocorneal synechiae, as well as Peters, Axenfeld, and Rieger anomalies. Here, we report a large five-generation Caucasian family exhibiting atypical syndromic ASD segregating with a novel truncating variant of FOXC1. The family history is consistent with highly variable autosomal dominant symptoms including isolated glaucoma, iris hypoplasia, aniridia, cataract, hypothyroidism, congenital heart anomalies, and cystic kidney disease. Whole exome sequencing revealed a novel variant [c.313_314insA; p.(Tyr105*)] in FOXC1 that disrupts the alpha-helical region of the DNA-binding forkhead box domain. In vitro studies using a heterologous cell system revealed aberrant cytoplasmic localization of FOXC1 harboring the Tyr105* variant, likely precluding downstream transcription function. Meta-analysis of the literature highlighted the intrafamilial variability related to FOXC1 truncating alleles. This study highlights the clinical variability in ASD and signifies the importance of combining both clinical and molecular analysis approaches to establish a complete diagnosis.
\end{abstract}

Keywords: FOXC1, in vitro studies, novel variant, ophthalmic genetics, intrafamilial variability, anterior segment dysgenesis

\section{Introduction}

Anterior segment dysgenesis (ASD) encompasses a wide spectrum of developmental abnormalities of the anterior ocular segment coupled with increased risk of glaucoma, Peters anomaly, iris hypoplasia, corectopia, or sclerocornea, which may lead to blindness [1]. Syndromic ASD is caused by specific genes, predominantly transcription factors and cofactors such as PAX6, PITX2, FOXC1, and BCOR [2-8], that are critical for the development of many tissues, including ocular, neuronal, craniofacial, dental, cardiac, and renal. Clinical heterogeneity, both phenotypic and genotypic, along with the overlapping of symptoms associated with anterior segment dysgenesis make the disease classification and clinical diagnosis challenging. This has been the case despite several attempts to categorize ASD to aid the diagnosis [9-11].

Forkhead box C1 (FOXC1) belongs to a family of transcription factor genes, and heterozygous variants have been associated with Axenfeld-Rieger syndrome and ASD [12-14]. FOXC1 plays a critical role during early development, specifically for the 
development of the anterior segment of the eye, including iris, lens, and cornea. Further, FOXC1 is involved in the normal development of the kidney, brain, and heart. More than 50 different variants in FOXC1 have been associated with Axenfeld-Rieger syndrome type 3. Variants may reduce the amount of functional FOXC1 that is produced or affect the DNA binding capability of FOXC1, resulting in functional haploinsufficiency, leading to variable ASD in humans and mice $[15,16]$. Variable expressivity associated with FOXC1 variants may be quite broad, with the observation of Peters anomaly, iris hypoplasia, or corectopia among family members harboring the same causal variant [13]. Here, we describe a novel truncating variant in FOXC1 causing autosomal dominant atypical ASD with highly variable phenotypic spectrum including variable iris hypoplasia and aniridia in a large Caucasian family. We also perform an extensive meta-analysis of the literature to explore the range and frequency of phenotypes related to FOXC1 truncating alleles segregating in families.

\section{Materials and Methods}

\subsection{Clinical Evaluation}

This study was conducted under institutional review board-approved protocols in accordance with the Declaration of Helsinki for the release of clinical information, family history, blood draw, and skin biopsies. Informed consent was obtained after the explanation of the study's risks and benefits.

\subsection{Whole exome sequencing (WES) and Sanger sequencing}

WES and data analysis were performed as described previously [17]. Exome sequencing was conducted on two affected individuals and was enriched using the Nimblegen SeqCap EZ Exome v2.0 Library (Roche Diagnostics; San Francisco, CA). Segregation analysis of the FOXC1 variant was performed using Sanger sequencing on the DNA samples of all of the participating family members.

\section{3. in vitro localization analysis}

We subcloned human FOXC1, a single coding exon gene, from genomic DNA samples, into tdTomato-C1 vector and mutant FOXC1 into GFP-C2 (Clonetech) with Bgl II and Sal I sites using infusion technology. Constructs were validated using Sanger sequencing. COS-7 cells were transfected with lipofectamine 2000, processed, and imaged using Zeiss 710 confocal microscope [18].

\subsection{Meta-analysis}

A literature review of intrafamilial variability in FOXC1-related disorders due to truncating variants was performed using The Human Gene Mutation Database (http://www.hgmd.cf.ac.uk/ac/index.php). Initially, a comprehensive review of 134 publically available reports of FOXC1-related disorder variants was performed to identify those with truncating variants $(n=41)$. Articles included in the final meta-analysis reported detailed phenotypes of multiple affected family members $(n=12)$ [[6], [14], 19-27].

\section{Results}

\subsection{Patient Characteristics}


The proband was a 38-year-old Caucasian female who presented with congenital glaucoma, bilateral iris hypoplasia, congenital cataracts, hypothyroidism, and mitral valve prolapse. She is part of the fourth generation of a five-generation family with a history of vision impairment along with multi-organ disorder. There were 12 affected individuals documented across five generations in this family, with glaucoma being the most predominant segregating phenotype. Out of the affected family members, seven were male and five were female. Numbered individuals (Figure 1a) in the family, across a range of ages (21-68 years), received a dilated eye exam as part of a comprehensive evaluation of visual function, and, based on clinical history, evaluation of cardiac and renal function.

\subsection{Clinical Examination}

Clinical data and blood samples for DNA extraction were obtained from seven family members with consent, and made available for this study. The family history is consistent with an autosomal dominant inheritance pattern presenting with highly variable symptoms including isolated glaucoma (12 out of the 25 family members), iris hypoplasia, aniridia, cataract, hypothyroidism, congenital heart anomalies, and cystic kidney disease (Figure 1a-b). Other individuals, such as the proband and the proband's mother (IV-2 and III-1, respectively), had a variation of phenotypes: ranging from cardiac valvular disease to iris hypoplasia to glaucoma.

\subsection{Genetic Analysis}

Whole exome sequencing was performed on two individuals, revealing a novel insertion variant (c.313_314insA) in FOXC1 (Figure 1c). This variant was predicted to lead to early protein truncation [p.(Tyr105*)] (Figure 1c-d). Additionally, the variant is predicted to disrupt the alpha-helical region of the DNA-binding forkhead box domain. Segregation of the variant along with disease phenotype was then found in four additional family members (Figure 1b), and was absent from an unaffected family member (Figure 1c). The highly variable phenotypes found in the assessed family spanned the entire spectrum of FOXC1-associated diseases and ranged from congenital glaucoma to Axenfeld-Rieger Syndrome (ARS). Axenfeld-Rieger Syndrome Type 3 is an autosomal dominant condition primarily characterized by developmental abnormalities of the anterior segment and is also associated with systemic abnormalities such as cardiac defects and hearing loss (OMIM ID: 602482). We found no pathogenic variants in other ASDassociated genes, which may explain the phenotypic variability within this family. 
a

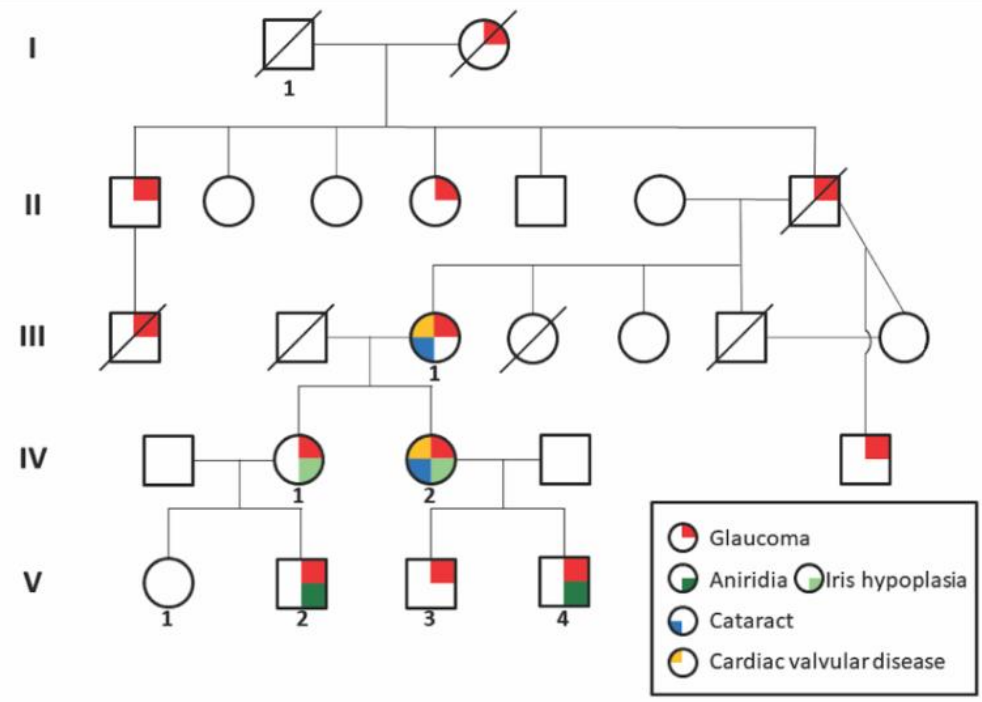

b

III-1

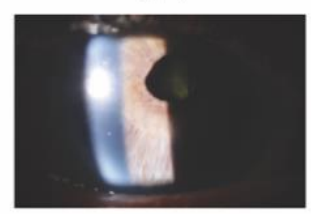

IV-1

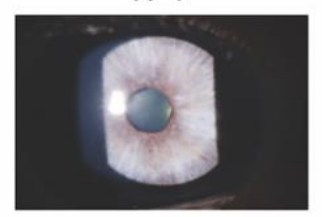

$\mathrm{V}-2$

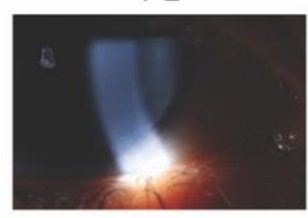

d

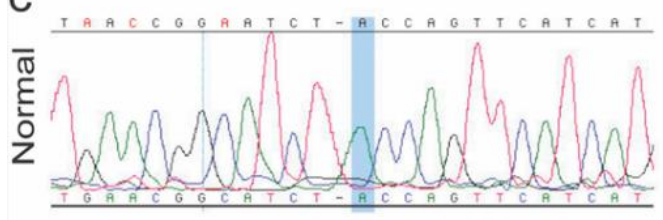

p.Tyr105*

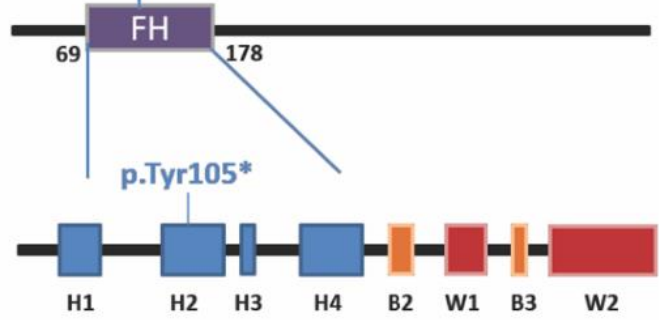

Figure 1. (a) Numbered individuals received a dilated eye examination. The predominant phenotype was congenital glaucoma, while other ocular and nonocular abnormalities exhibited variable expression; (b) Slit lamp analysis revealed multiple eye problems. The proband (IV-1) is a 38-yearold female with congenital glaucoma, bilateral iris hypoplasia, congenital cataracts, hypothyroidism, and mitral valve prolapse. Family history is consistent with a highly variable autosomal dominant condition that includes isolated glaucoma (III-01), iris hypoplasia (IV-01), aniridia (V-02), cataract, hypothyroidism, congenital heart anomalies, and cystic kidney disease; (c) Representative Sanger sequencing chromatograms of FOXC1 exon from a normal (V-1) and an affected (V-2) individuals of family LNG5, showing the c.313_314insA allele. All individuals included within the LNG5 pedigree were tested with Sanger sequencing to confirm segregation and full phenotypic penetrance; (d) Schematic representation of FOXC1 gene and protein structure. Two nuclear localization signals (NLS1 and NLS2) are present at both ends of the DNA-binding forkhead box domain (FH). FOXC1 c.313_314insA [p.(Tyr105*)] disrupts the alpha-helical region of the FH domain and removes NLS2 along with the carboxy tail. 
Next, to assess the functional impact of the novel FOXC1 variant, we generated fluorescently tagged constructs, tdTomato-FOXC1WT and GFP-FOXC1p.Tyr105*. Transient transfection in COS-7 cells showed that FOXC1 ${ }^{\text {WT }}$ localized exclusively in the nucleus as expected. The p.Tyr105* is positioned in between two known nuclear localization sequences, NLS1 and NLS2. Hence, the variant-mediated early truncation was predicted to eliminate NLS2. Corresponding to the in silico prediction, overexpression of FOXC1 ${ }^{\text {Tyr105* }}$ in COS-7 cells revealed that the variant protein was partially localized in the cytoplasm (Figure 2), as opposed to the exclusive nuclear localization of WT protein. The mislocalization of FOXC1 $1^{\text {Tyr } 105^{*}}$ protein may likely disrupt its downstream transcriptional function. Overall, our in vitro studies suggets that both NLS1 and NLS2 motifs are essential for FOXC1 nuclear localization.

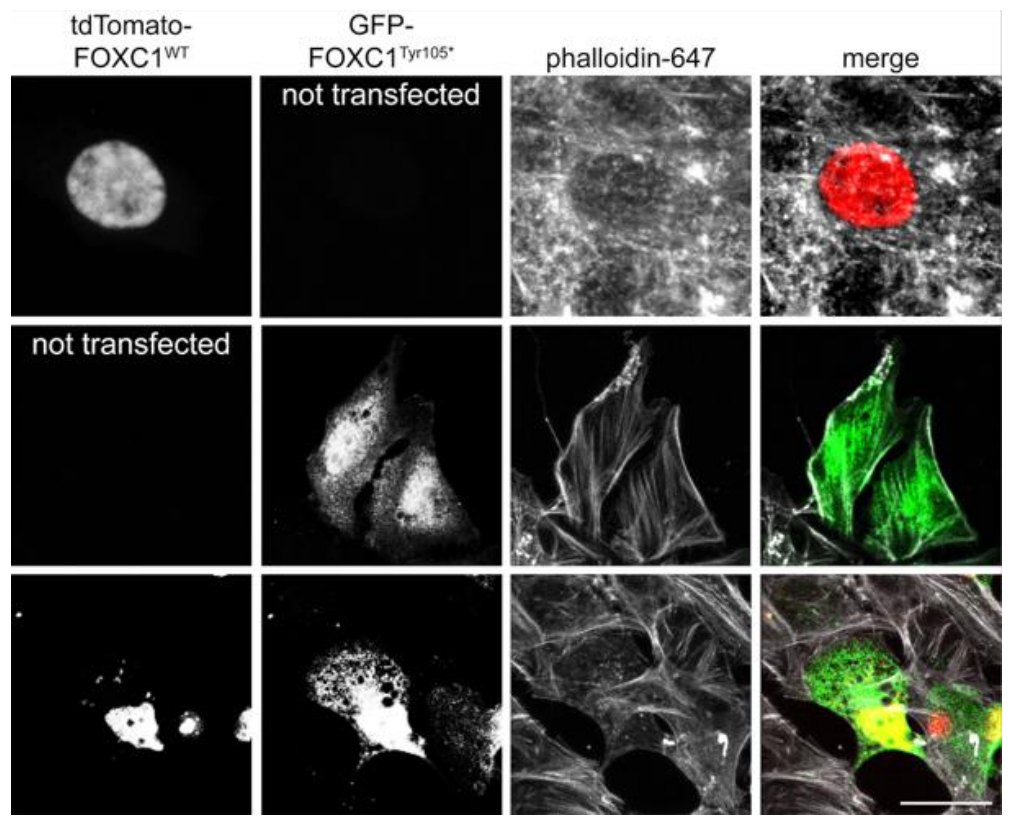

Figure 2. Representative images of COS-7 cells transiently transfected with tomato-tagged wildtype (WT) or FOXC1 constructs. As compared to WT-FOXC1, which is primarily localized in the nucleus, the truncated FOXC1 was also found in the cytoplasm. Scale bar $-10 \mu \mathrm{m}$. 
Table 1.

\begin{tabular}{|c|c|c|c|c|c|}
\hline DNA change & Amino acid change & Classification & Proband Phenotype & Intrafamilial Phenotypes & Reference \\
\hline c.15delC & p.(Arg4Hisfs*43) & Frameshift & Congenital glaucoma, optic atrophy & $\begin{array}{c}2 \text { member, } 2 \text { generations; optic atrophy, } \\
\text { unilateral superior oblique palsy }\end{array}$ & [19] \\
\hline c. $67 \mathrm{C}>\mathrm{T}$ & p. $\left(G \ln 23^{*}\right)$ & Nonsense & $\begin{array}{c}\text { Posterior embryotoxon, iris hypoplasia, ocular } \\
\text { hypertelorism, flat midface, microdontia, protu- } \\
\text { berant umbilical skin }\end{array}$ & $\begin{array}{l}9 \text { members, } 3 \text { generations; posterior embryo- } \\
\text { toxon, iris hypoplasia, glaucoma, flat midface, } \\
\text { microdontia, ocular hypertelorism, protuberant } \\
\text { umbilical skin, hearing loss, cardiac defect }\end{array}$ & [20] \\
\hline c.317delA & p.(Gln106Argfs*75) & Frameshift & $\begin{array}{c}\text { Glaucoma, iridocorneal adhesion, iris atrophy, } \\
\text { Haab's striae, iris hypoplasia, ocular hyper- } \\
\text { telorism, telecanthus, flat face, flat broad nasal } \\
\text { bridge }\end{array}$ & $\begin{array}{l}4 \text { members, } 2 \text { generations; corectopia, posterior } \\
\text { embryotoxon, elevated IOP, glaucoma, iridocor- } \\
\text { neal adhesion, iris hypoplasia, iris atrophy, flat } \\
\text { midface, flat broad nasal bridge, normal intelli- } \\
\text { gence }\end{array}$ & [21] \\
\hline c.313_314insA & p.(Tyr105*) & Frameshift & $\begin{array}{c}\text { Congenital glaucoma, bilateral iris hypoplasia, } \\
\text { congenital cataracts, hypothyroidism, mitral } \\
\text { valve prolapse }\end{array}$ & $\begin{array}{l}12 \text { members, } 5 \text { generations; glaucoma, iris hypo- } \\
\text { plasia, aniridia, cataract, hypothyroidism, con- } \\
\text { genital heart anomalies, cystic kidney disease }\end{array}$ & $\begin{array}{l}\text { Present } \\
\text { Study }\end{array}$ \\
\hline c. $358 \mathrm{C}>\mathrm{T}$ & p. $\left(\mathrm{G} \ln 120^{*}\right)$ & Nonsense & $\begin{array}{l}\text { Displaced Schwalbe line, iridocorneal adhesion, } \\
\text { iris hypoplasia, glaucoma, leukoma, maxillary } \\
\text { hypoplasia, ureteral stenosis }\end{array}$ & $\begin{array}{l}7 \text { members, } 3 \text { generations; posterior embryo- } \\
\text { toxon, iridocorneal adhesion, iris hypoplasia, } \\
\text { corectopia, glaucoma, maxillary hypoplasia, oc- } \\
\text { ular hypertelorism, atrial septal defect, ureteral } \\
\text { stenosis, protuberant umbilical skin }\end{array}$ & [22] \\
\hline c. $367 \mathrm{C}>\mathrm{T}$ & p. $\left(\mathrm{G} \ln 123^{*}\right)$ & Nonsense & $\begin{array}{l}\text { Corneal haze, elevated IOP with normal cup to } \\
\text { disc ratio }\end{array}$ & $\begin{array}{l}4 \text { members ( } 3 \text { affected, } 1 \text { unaffected with vari- } \\
\text { ant), } 3 \text { generations; secondary glaucoma, } \\
\text { phthisis bulbi, total cupping }\end{array}$ & [14] \\
\hline c.437_453del17 & p.(Pro146Alafs*85) & Frameshift & $\begin{array}{l}\text { Glaucoma, posterior embryotoxon, iris hypo- } \\
\text { plasia, early-onset glaucoma, hearing loss, ocu- } \\
\text { lar hypertelorism, telecanthus, megalocornea }\end{array}$ & $\begin{array}{c}2 \text { members, } 2 \text { generations; posterior embryo- } \\
\text { toxon, iris hypoplasia, corectopia, early-onset } \\
\text { severe glaucoma, atrial septal defect, aortic ste- } \\
\text { nosis, pulmonary stenosis, hearing loss, ocular } \\
\text { hypertelorism, telecanthus }\end{array}$ & [23] \\
\hline c. $456 \mathrm{G}>\mathrm{A}$ & p.(Trp152*) & Nonsense & $\begin{array}{l}\text { Childhood-onset glaucoma with normal IOP } \\
\text { and visual acuity, posterior embryotoxon, pe- } \\
\text { ripheral iridocorneal adherences, iris hypo- } \\
\text { plasia, short stature, maxillary hypoplasia, den- } \\
\text { tal abnormalities } \\
\end{array}$ & $\begin{array}{l}6 \text { members, } 3 \text { generations; developmental glau- } \\
\text { coma, posterior embryotoxon, peripheral irido- } \\
\text { corneal adhesion, iris hypoplasia, short stature, } \\
\text { maxillary hypoplasia, dental abnormalities, }\end{array}$ & [6] \\
\hline c. $477 \mathrm{C}>\mathrm{G}$ & p.(Tyr159*) & Nonsense & $\begin{array}{l}\text { Abnormal eye development (unspecified), leu- } \\
\text { koencephalopathy, global developmental delay, } \\
\text { hyperreflexia }\end{array}$ & $\begin{array}{l}3 \text { members, } 2 \text { generations; abnormal eye devel- } \\
\text { opment (unspecified), leukoencephalopathy }\end{array}$ & [24] \\
\hline
\end{tabular}
hyperreflexia 
c.609delC

p.(Ala204Argfs*111)

Corneal vascularization, thickened cornea (bilat-

eral corneal opacity with peripheral corneal vascularization), corneal perforation, iris hypo-

plasia, posterior embryotoxon, iris heterochromia, whole globe abnormalities (unspecified),

lens extrusion, persistent hyperplastic primary vitreous, retinal detachment, anterior segment developmental abnormalities, elevated IOP,

Descemet's membrane, absent endothelium,

\begin{tabular}{lcccc}
\hline c.1193_1196dup & p.(Met400Serfs*129) & Frameshift & $\begin{array}{c}\text { Congenital glaucoma, microphthalmia, iris } \\
\text { anomalies, pupil anomalies, dysmorphic facial } \\
\text { features, bilateral club foot, mild cognitive im- } \\
\text { pairment, congenital deafness }\end{array}$ & $\begin{array}{c}\text { 2 members, 2 generations; non-congenital glau- } \\
\text { coma, hearing loss, club foot }\end{array}$ \\
\hline c.1265C $>$ A & p.(Ser422*) & Nonsense & $\begin{array}{c}\text { Posterior embryotoxon, corectopia, megalocor- } \\
\text { nea, ocular hypertension, normal systemic fea- } \\
\text { tures }\end{array}$ & $\begin{array}{c}2 \text { members, } 1 \text { generation; glaucoma, no other } \\
\text { ocular features, normal systemic features }\end{array}$ \\
\hline
\end{tabular}

*Note: All nonsense truncating alleles have zero minor allele frequency in the gnomAD database. 566 total amino acids in FOXC1. IOP (intraocular pressure); fs - frameshift. mostly absent Bowman's layer, absent

$$
\text { central corneal cyst }
$$

3 members, 2 generations; iris hypoplasia, posterior embryotoxon, iris heterochromia, peripheral iridocorneal adhesions, down-slanting palpebral fissures, ocular hypertelorism, malar hypoplasia, mild retrognathia, unusual dentition requency in the gnom 


\subsection{Literature Meta-Analysis}

To further explore FOXC1-related intrafamilial variability, we performed a comprehensive literature review of early truncation alleles of FOXC1 that segregated in multiple affected family members with available reported clinical phenotypes (Table 1; $n$ = 12 publication, 56 affected individuals). A majority of the segregating variants were found across multiple generations (11/12). Similar to our study, we found extensive variability among intrafamilial phenotypes due to truncating variants (Table 1). All of the families had individuals who could be categorized as "more severe" due to increased severity and breadth of disease presentation, and individuals that could be categorized as "less severe" due to the isolated presentation of phenotypes. The variation in the range of intrafamilial disease severity is a general phenomenon among genetically inherited diseases, particularly prominent in ocular and systemic diseases. Overall, only one family had affected individuals with only nonsyndromic presentations (normal systemic features), five families with affected individuals who all had syndromic presentations, and six families with affected individuals that had either syndromic and nonsyndromic presentations. This supports the fact that ASD cannot be ruled out in cases with nonsyndromic presentations and further clinical evaluation is recommended.

While Axenfeld-Rieger anomaly was listed as a key clinical diagnosis for 9 out of 12 of the FOXC1 variants that are listed in Table 1, it does not capture the range of ocular phenotypes that were observed in these families. $63 \%$ of affected individuals from Table 1 were affected with glaucoma and 61\% were affected with ASD (Figure 3). Additionally, the range of ocular phenotypes include optic atrophy, Haab striae, iridocorneal adhesion, iris atrophy, corectopia, posterior embryotoxon, elevated intraocular pressure, aniridia, displaced Schwalbe line, corneal haze, phthisis bulbi, thickened cornea, iris heterochromia, corneal perforation, lens extrusion, retinal detachment, hyperplastic primary vitreous, abnormalities of the Bowman's layer, abnormalities of the Descemet membrane, abnormalities of the corneal endothelium, corneal cysts, and megalocornea.

Further, differences in the frequency of FOXC1-associated phenotypes were noted. While relatively commonly occurring phenotypes are depicted in Figure 3, a significant number of features were not common and some features were only unqiue to singular families. Surprisingly, aniridia was not reported to be associated with FOXC1 in any of the reviewed publications and to the best of our knowledge is a unique feature of the SNP associated with the FOXC1-affected family reported in this study. Moreover, common systemic abnormalities such as hearing impairment and cardiac-related abnormalities were less common, only seen in $10 \%$ and $7 \%$ of the studied affected individuals found in the meta-analysis, respectively. 


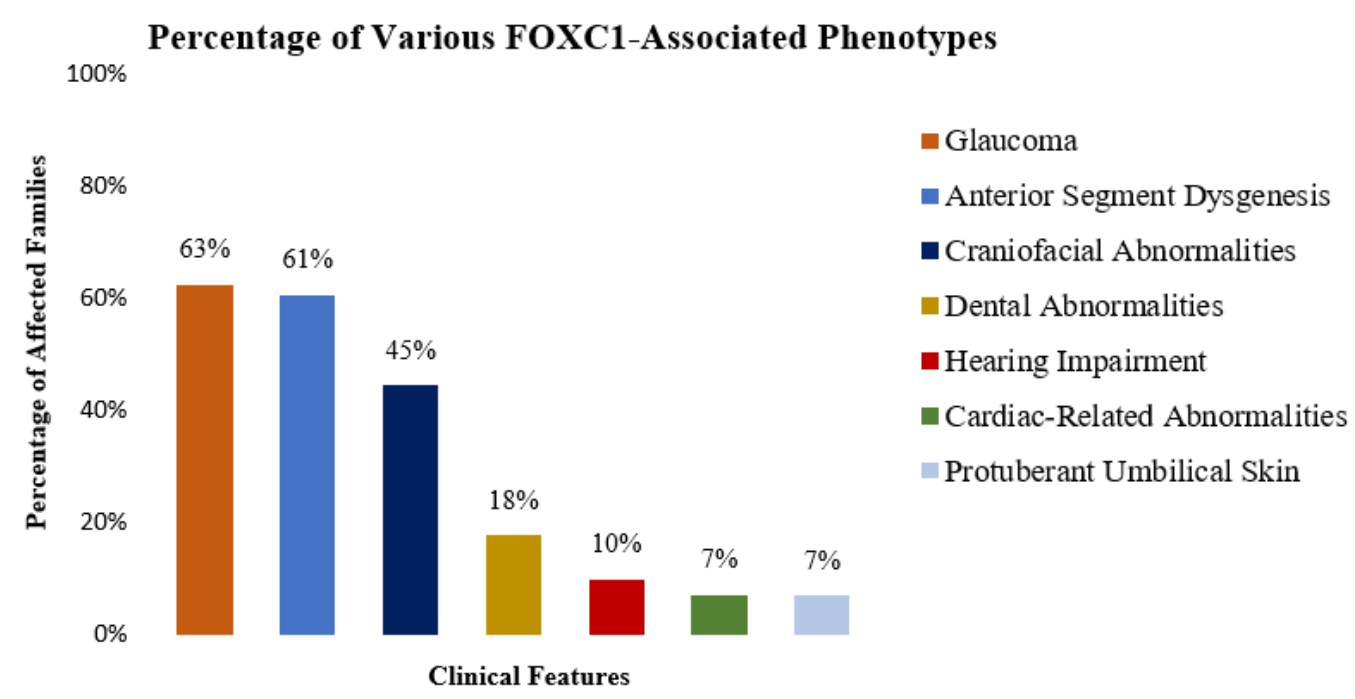

Figure 3. Prevalence of the commonly occurring clinical features among 56 individuals from 12 families affected with truncating variants of FOXC1 from Table 1 ( $n=56$ individuals).

\section{Discussion}

Here, we performed a genetic analysis on seven individuals from a large (12 of 25 family members were affected), multigenerational family.and we found a novel nonsense variant in FOXC1 causing extensive variable phenotypic expressivity among family members, including variable iris hypoplasia and aniridia. Aniridia is a very uncommon FOXC1-related phenotype. We observed intrafamilial clinical heterogeneity proband (IV:2) presented with severe syndromic presentation including glaucoma, iris hypoplasia, cataract, and cardiac valvular disease, while other family members with the same variant had milder phenotypic presentations. IV-1 (sister of proband), only presented with iris hypoplasia and glaucoma.

Meta-analysis of early truncation allaes of FOXC1-associated phenotypes revealed extensive intrafamilial and interfamilial heterogeneity of ocular and systemic phenotypes in 12 families. Variants in the PAX6 gene account for $>90 \%$ of all aniridia cases, regardless of whether they are familial ( $\sim 66 \%)$ or sporadic ( $33 \%)$ [28]. Previously, only one variant in FOXC1 (c.454T>G; p.Trp152Gly) has been associated with aniridia [29]. We postulate that FOXC1-related aniridia appears to be the extreme end of iris hypoplasia rather than true aplasia as is seen in PAX6-related aniridia [30].

The phenomes from the meta-analysis present a variety of ocular abnormalities ranging from Haab's striae to cataract to microphthalmia with pronounced intrafamilial variability. In reference 27 , the proband exhibited posterior embryotoxon, corectopia, megalocornea, and ocular hypertension but the affected sibling was only reported to have isolated glaucoma. Additionally, one proband was affected with a range of ocular abnormalities: glaucoma, microphthalmia, iris anomalies, and pupil anomalies, whilethe assessed parent presented with only glaucoma [27]. Often, the phenotypic presentation of the proband was the most severe among family members, which is not surprising since the proband prompted the genetic investigation. 
Review of the meta-analysis revealed additional variability among systemic features related to FOXC1. While the predominant abnormalities were ocular, FOXC1related abnormalities also extended into other organ systems. Three families reported having afflictions related to the cardiac system including the family reported in this study [[20], [23]]. There were three families reported to have hearing impairments [[20], [23], [26]]. Interestingly, protuberant umbilical skin, considered a salient feature for FOXC1-related ARS, was only noted in two families. Two families were noted to have an affliction related to the genitourinary system: cystic kidney disease (in the present study) and ureteral stenosis [22]. Global developmental delay was noted in only one family. However, not all of these phenotypes have a causally established association with a FOXC1 variant. While we cannot exclude the possibility that certain phenotypes are due to a secondary causes, such as a congenital malformation or a second Mendelian disease, the recurrent observations of systemic findings in similar tissues systems warrants consideration of systemic evaluations in all family members with FOXC1-related disorders.

The meta-analysis portion of our study further highlights the broad spectrum of phenotypes associated with FOXC1, both in the variability of tissues affected and their clinical co-morbidities. Surprisingly, the presentations that extended past ophthalmological afflictions into other organ systems were less common than expected. This is possibly due to incomplete inclusion of all clinical details or differences in systemic investigations performed in different reports across time, which is a limitation of a literature-based meta-analyses such as this. As such, the list of phenotypes described in Table 1 and Figure 3 may be underrepresented. However, our findings do recommend comprehensive ocular and systemic examinations, even when other family members are determined to have partial phenotypic expression of FOXC1-related disorders.

Despite the extensive phenotypic variability observed in individuals harboring FOXC1 variants, the underlying mechanism of variability is currently unknown. Environmental factors (e.g. drug exposure), reduced penetrance at the tissue level, subclinical phenotypic features, and/or genetic modifiers may partially explain the clinical variability. Future studies with a larger sample size would be required to test these rigourously.

In summary, our study further affirms the variability among individuals and families with FOXC1-related ARS, as well as diagnostic and testing challenges. ASD should be considered in apparently isolated congenital glaucoma or cataracts. The additional risk of congenital cardiac and renal disease should also be taken into consideration where ASD is noted. Therefore, the combination of systemic clinical and molecular analysis is likely the most efficient approach to establish a diagnosis and complete a systemic evaluation of ASD.

Author Contributions: Conceptualization, R.B.H.; methodology, M.R.A., S.S., M.B.Y. and R.B.H.; validation, S.S., and M.R.A.; formal analysis, M.B.Y. and R.B.H.; investigation, S.S.; M.R.A.; and M.B.Y.; resources, M.B.Y. and R.B.H.; data curation, S.S. M.R.A. and M.B.Y.; writing-original draft preparation, S.S. M.R.A. and R.B.H.; writing-review and editing, M.B.Y., S.S.; supervision, R.B.H.; project administration, M.B.Y. and R.B.H.; funding acquisition, R.B.H. All authors have read and agreed to the published version of the manuscript. 
Funding: This research was funded by THE NATIONAL EYE INSTITUTE INTRAMURAL FUNDS, grant number ZIAEY000564, ZIAEY000565.

\section{Institutional Review Board Statement:}

The study was conducted in accordance with the Declaration of Helsinki and approved by the Institutional Review Board (or Ethics Committee) of Cincinnati Children Hospital Medical Hospital, Cincinnati (protocol code 2010-0452 and July 1st, 2012).

Informed Consent Statement: Informed consent was obtained from all subjects involved in the study after the explanation of the study risks and benefits.

Data Availability Statement: Planned submission to ClinVar

Acknowledgments: We thank Thomas Jaworek for his technical assistance that contributed to the success of this project.

Conflicts of Interest: The authors declare that they have no conflict of interest. 


\section{References}

1. Reis, L.M. and E.V. Semina, Genetics of anterior segment dysgenesis disorders. Current opinion in ophthalmology, 2011. 22(5): p. 314-324.

2. Berry, F.B., et al., Functional interactions between FOXC1 and PITX2 underlie the sensitivity to FOXC1 gene dose in Axenfeld-Rieger syndrome and anterior segment dysgenesis. Hum Mol Genet, 2006. 15(6): p. 905-19.

3. Borges, A.S., et al., Genetic analysis of PITX2 and FOXC1 in Rieger Syndrome patients from Brazil. J Glaucoma, 2002. 11(1): p. 51-6.

4. Wang, Y., et al., Novel identification of a four-base-pair deletion mutation in PITX2 in a Rieger syndrome family. J Dent Res, 2003. 82(12): p. 1008-12.

5. Chavarria-Soley, G., et al., Novel CYP1B1 and known PAX6 mutations in anterior segment dysgenesis (ASD). J Glaucoma, 2006. 15(6): p. 499-504.

6. Cella, W., et al., Structural assessment of PITX2, FOXC1, CYP1B1, and GJA1 genes in patients with AxenfeldRieger syndrome with developmental glaucoma. Invest Ophthalmol Vis Sci, 2006. 47(5): p. 1803-9.

7. Du, R.F., et al., A Novel Mutation of FOXC1 (R127L) in an Axenfeld-Rieger Syndrome Family with Glaucoma and Multiple Congenital Heart Diseases. Ophthalmic Genet, 2014: p. 1-5.

8. Kapoor, S., et al., Dysmyelination of the cerebral white matter with microdeletion at 6p25. Indian Pediatr, 2011. 48(9): p. 727-9.

9. Alward, W.L., Axenfeld-Rieger syndrome in the age of molecular genetics. Am J Ophthalmol, 2000. 130(1): $p$. 107-15.

10. Ito, Y.A. and M.A. Walter, Genomics and anterior segment dysgenesis: a review. Clinical \& Experimental Ophthalmology, 2014. 42(1): p. 13-24.

11. Idrees, F., et al., A Review of Anterior Segment Dysgeneses. Survey of Ophthalmology, 2006. 51(3): p. 213-231.

12. Tumer, Z. and D. Bach-Holm, Axenfeld-Rieger syndrome and spectrum of PITX2 and FOXC1 mutations. Eur J Hum Genet, 2009. 17(12): p. 1527-39.

13. Honkanen, R.A., et al., A family with Axenfeld-Rieger syndrome and Peters Anomaly caused by a point mutation (Phe112Ser) in the FOXC1 gene. Am J Ophthalmol, 2003. 135(3): p. 368-75.

14. Komatireddy, S., et al., Mutation spectrum of FOXC1 and clinical genetic heterogeneity of Axenfeld-Rieger anomaly in India. Mol Vis, 2003. 9: p. 43-8.

15. Smith, R.S., et al., Haploinsufficiency of the transcription factors FOXC1 and FOXC2 results in aberrant ocular development. Hum Mol Genet, 2000. 9(7): p. 1021-32.

16. Nishimura, D.Y., et al., A spectrum of FOXC1 mutations suggests gene dosage as a mechanism for developmental defects of the anterior chamber of the eye. Am J Hum Genet, 2001. 68(2): p. 364-72.

17. Jaworek, T.J., et al., An Alteration in ELMOD3, an Arl2 GTPase-Activating Protein, Is Associated with Hearing Impairment in Humans. PLOS Genetics, 2013. 9(9): p. e1003774.

18. Sethna, S., et al., Regulation of Phagolysosomal Digestion by Caveolin-1 of the Retinal Pigment Epithelium Is Essential for Vision. Journal of Biological Chemistry, 2016. 291(12): p. 6494-6506.

19. Chakrabarti, S., et al., The transcription factor gene FOXC1 exhibits a limited role in primary congenital glaucoma. Invest Ophthalmol Vis Sci, 2009. 50(1): p. 75-83.

20. Mirzayans, F., et al., Axenfeld-Rieger syndrome resulting from mutation of the FKHL7 gene on chromosome 6p25. Eur J Hum Genet, 2000. 8(1): p. 71-4. 
21. Kim, G.N., et al., A novel forkhead box C1 gene mutation in a Korean family with Axenfeld-Rieger syndrome. Mol Vis, 2013. 19: p. 935-43.

22. Weisschuh, N., et al., A novel mutation in the FOXC1 gene in a family with Axenfeld-Rieger syndrome and Peters' anomaly. Clin Genet, 2008. 74(5): p. 476-80.

23. Fuse, N., et al., Novel mutations in the FOXC1 gene in Japanese patients with Axenfeld-Rieger syndrome. Mol Vis, 2007. 13: p. 1005-9.

24. Kumar, M., C. Chambers, and R. Dhamija, Axenfeld-Rieger Syndrome and Leukoencephalopathy Caused by a Mutation in FOXC1. Pediatr Neurol, 2017. 66: p. 113-114.

25. Kelberman, D., et al., Digenic inheritance of mutations in FOXC1 and PITX2 : correlating transcription factor function and Axenfeld-Rieger disease severity. Hum Mutat, 2011. 32(10): p. 1144-52.

26. Reis, L.M., et al., Whole exome sequencing identifies multiple diagnoses in congenital glaucoma with systemic anomalies. Clin Genet, 2016. 90(4): p. 378-82.

27. Souzeau, E., et al., Glaucoma spectrum and age-related prevalence of individuals with FOXC1 and PITX2 variants. Eur J Hum Genet, 2017. 25(7): p. 839-847.

28. Axton, R., et al., The incidence of PAX6 mutation in patients with simple aniridia: an evaluation of mutation detection in 12 cases. Journal of Medical Genetics, 1997. 34(4): p. 279.

29. Ito, Y.A., et al., Severe Molecular Defects of a Novel FOXC1 W152G Mutation Result in Aniridia. Investigative Ophthalmology \& Visual Science, 2009. 50(8): p. 3573-3579.

30. Souzeau, E., et al., PAX6 molecular analysis and genotype-phenotype correlations in families with aniridia from Australasia and Southeast Asia. Mol Vis, 2018. 24: p. 261-273. 